\title{
HERTZSPRUNG'S LUMINOSITY-COLOUR RELATION FOR VISUAL BINARIES
}

\author{
By W. S. Finsen ANd P. N. J. Wisse
}

Johannesburg, South Africa

The number of visual binaries for which orbits have been computed now exceeds 600 , but this laboriously accumulated wealth of data remains relatively sterile owing to the paucity of reliable parallaxes. Hertzsprung (1964) had this in mind when he revived the idea he first voiced in 1911 and proposed a new luminosity parameter defined as the absolute magnitude of a star reduced to unit mass without changing its density or surface brightness. The great advantage of this parameter is the fact that it is readily derived from the period and semi-major axis of a visual binary without recourse to the parallax. It therefore permits immediate use of most of the computed orbits for the construction of a luminosity-colour diagram analogous to the classical H-R diagram but entirely free of the scatter resulting from errors in the parallaxes.

Hertzsprung called the new parameter "la luminosité de masse" or "massIuminosity" and denoted it by $m_{L}$, but we feel that this is likely to lead to confusion with the mass-luminosity relation. We propose, therefore, the name 'specific magnitude' and denote it by $M_{s}$. We also adopt the more usual definition of absolute magnitude as the magnitude at a distance of ten parsecs rather than one parsec as preferred by Hertzsprung.

Let the mass of a star be denoted by $\mathfrak{M}$, its absolute magnitude by $M_{v}$, and its specific magnitude, defined as above, by $M_{s}$. Then the ratio of the surface areas of two stars of mass unity and $\mathfrak{M}$ respectively, and of the same density, is $\mathfrak{M}^{-2 / 3}$. If both have the same surface brightness this corresponds to a difference of magnitude of $5 / 3 \log \mathfrak{M}$, and therefore

$$
M_{s}-M_{v}=\frac{5}{3} \log \mathfrak{M} \text {. }
$$

For a single star, therefore, the specific magnitude may be derived from the absolute magnitude through the intermediary of the mass-luminosity relation.

For a double star we have

$$
\mathfrak{M}_{A}+\mathfrak{M}_{B}=a^{3} p^{-3} P^{-2}
$$

where $a$ is the semi-major axis and $p$ the parallax in seconds of arc and $P$ is the period in years. Equation (1) may be written, for the brighter component $A$,

$$
M_{s, A}=m_{A}+5+5 \log p+\frac{5}{3} \log \mathfrak{M}
$$

R.A.S.C. Jour., Vol. 67, No. 2 
where $m_{A}$ is the apparent magnitude. On eliminating the parallax $p$ from equations (2) and (3) we have

(4) $M_{s, A}=m_{A}+5+5 \log a-\frac{10}{3} \log P+\frac{5}{3} \log \left\{\mathfrak{M}_{A} /\left(\mathfrak{M}_{A}+\mathfrak{M}_{B}\right)\right\}$

which may be written

$$
M_{s, A}=m_{A}+5+5 \log h_{1}+\frac{5}{3} \log \left\{\mathfrak{M}_{A} /\left(\mathfrak{M}_{A}+\mathfrak{M}_{B}\right)\right\}
$$

and for the fainter component $B$

$$
M_{s, B}=m_{B}+5+5 \log h_{1}+\frac{5}{3} \log \left\{\mathfrak{M}_{B} /\left(\mathfrak{M}_{A}+\mathfrak{M}_{B}\right)\right\}
$$

where

$$
\begin{aligned}
5 \log h_{1} & =5 \log a-\frac{10}{3} \log P & & \text { for elliptic orbits, } \\
& =5 \log q+\frac{10}{3} \log n-2.159 & & \text { for parabolic orbits, } \\
& =5 \log a+\frac{10}{3} \log n-2.661 & & \text { for hyperbolic orbits. }
\end{aligned}
$$

For both parabolic and hyperbolic orbits $q$ is the periastron distance in the true orbit in seconds of arc. For parabolic orbits $n=\sigma / q^{2}$ and for hyperbolic orbits

$$
n=\frac{2 \sigma(e-1)^{2}}{q^{2}\left(e^{2}-1\right)^{\frac{1}{2}}}
$$

where $\sigma$ is the areal constant in the true orbit in square seconds of arc per annum.

It will be noted, incidentally, that $h_{1}$ is the so-called "hypothetical" parallax corresponding to $\mathfrak{M}_{A}+\mathfrak{M}_{B}=1$.

For pairs with small difference of magnitude we may, following Hertzsprung, assume $\mathfrak{M}_{A}=\mathfrak{M}_{B}$ and obtain a mean value of the specific magnitude of the two components, i.e.,

$$
M_{s}=m_{A+B}+5+5 \log h_{1}+0.25 \text {. }
$$

Hertzsprung confined his attention to such pairs, noting only that with unequal pairs one may determine $M_{s}$ for each component if one assumes a relation between $\Delta m$ and $\Delta \log \mathfrak{M}$.

Assuming that $\Delta \log \mathfrak{M}=k \Delta m$ we have readily for the general case 


$$
\begin{gathered}
M_{s, A}=m_{A}+5+5 \log h_{1}-\frac{5}{3} \log \left(1+10^{k \Delta m}\right) \\
M_{s, B}=m_{B}+5+5 \log h_{1}-\frac{5}{3} \log \left(1+10^{-k \Delta m}\right)
\end{gathered}
$$

where $\Delta m=m_{B}-m_{A}$, is always positive.

The empirical mass-luminosity relation of Harris, Strand, and Worley (1963) gives $k \cong-0.10$ for stars brighter than 7.5 absolute bolometric magnitude, and $k \cong-0.14$ for fainter stars. For our calculations we have used the following values:

$$
\begin{array}{ll}
\text { spectral type earlier than } \mathrm{K} 0: & k=-0.10 \\
\text { spectral type } \mathrm{K} 0 \text { to } \mathrm{K} 9: & k=-0.12 \\
\text { spectral type later than } \mathrm{K} 9: & k=-0.14
\end{array}
$$

Should it be desired to convert $M_{s}$ into $M_{v}$, this may be done by means of equation (1) and the mass-luminosity relation. Using the relation of Harris, Strand, and Worley quoted above we obtain

$$
\begin{aligned}
M_{v} & =1.20 M_{s}+0.20(\mathrm{BC})-0.92 & & \left(M_{b o l}<+7.5\right) \\
& =1.32 M_{s}+0.32(\mathrm{BC})-1.66 & & \left(M_{b o l}>+7.5\right)
\end{aligned}
$$

where $\mathrm{BC}$ is the bolometric correction.

The absolute magnitude $M_{v}$ (like the dynamical parallax) depends of course directly on the mass-luminosity relation, but equations (8) show that the specific magnitude $M_{s}$ depends only on the derivative of the massIuminosity relation and is completely independent of it when the difference of magnitude is zero. For moderate differences of magnitude the dependence is not critical; for example, a difference of magnitude of 1.0 affects the specific magnitudes of both components by only 0.1 .

Equations (8) were used for computing specific magnitudes from the data in the orbit catalogue of Finsen and Worley (1970). Grade 5 and hyperbolic orbits were excluded, as also were systems with variable or white dwarf components, or with components definitely known to be composite. In most cases, when two or more orbits were catalogued, the corresponding values of $M_{s}$ were sufficiently accordant for the mean to be adopted, even in the case of widely-differing "ambiguous" orbits resulting from uncertainty of quadrant interpretation; otherwise they were excluded. Figure 1 shows the specific magnitudes plotted against the colour indices $B-V$ taken from the photoelectric catalogue of Blanco, Demers, Douglass, and FitzGerald (1968) or from the lists of Smak (1967), Knipe (1969, 1970), and Alexander (1970). Most of the values plotted refer to the brighter components, but in a few cases $B-V$ was available also for the fainter compo- 


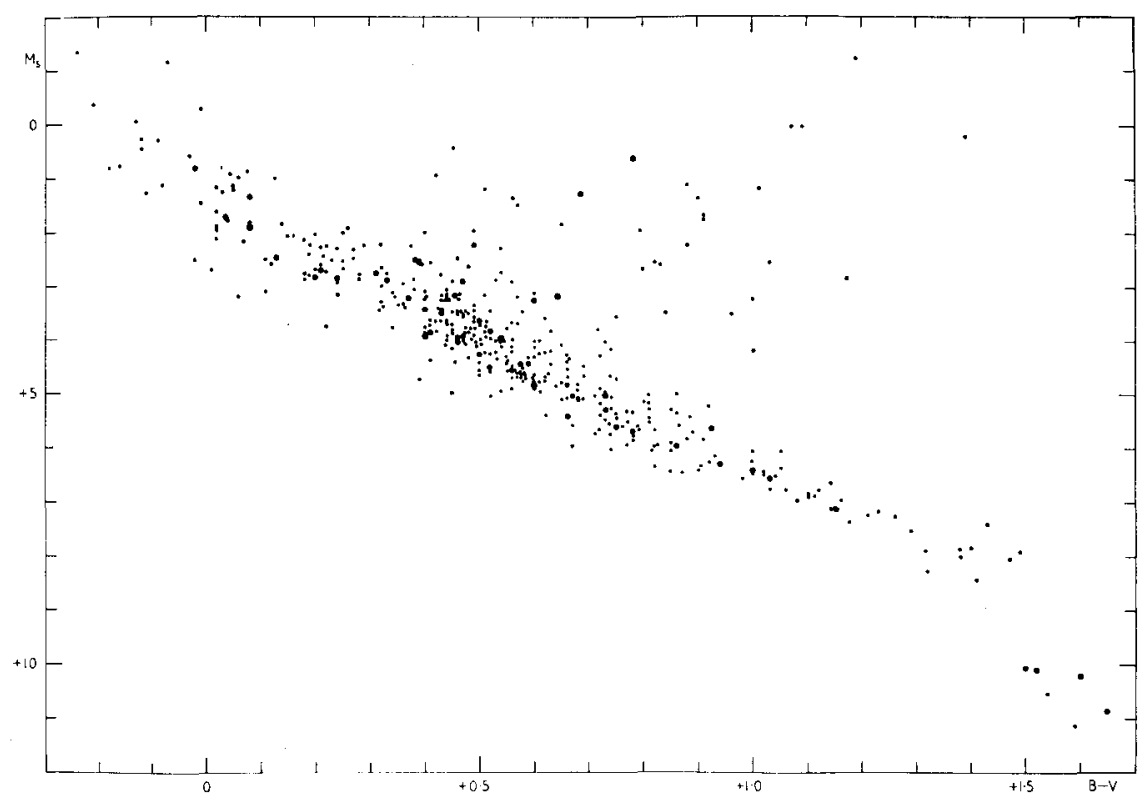

FIG. 1. The relation between specific magnitude, $M_{B}$, and colour index, $B-V$, for components of visual binaries. The larger dots indicate values based on grade 1 orbits.

nents, enabling both to be plotted. The total number of systems represented in the diagram is $407 ; 142$ other systems lacked colour-index determinations and, therefore, could not be used.

Values of $M_{s}$ derived from the most reliable orbits (grade 1) are identified in the diagram by the use of larger dots. It will be noticed that the scatter exhibited by them is practically of the same order as for the rest of the material, indicating that it is largely intrinsic rather than due to accidental errors. This is confirmed by examining individual deviant values based on grade 1 orbits; in no case was it found possible to account for the deviation by assuming plausible corrections to the orbital or other data. Observational errors in $B-V$ are not likely to contribute greatly to the scatter, although in one case the value of $M_{s}$ showed a marked deviation from the main sequence, but this was traced to a misprint in the catalogued value of $B-V$ (see Table II). As a matter of fact, the values of $M_{s}$ yielded by "indeterminate" (grade 5 ) orbits and even hyperbolic orbits showed scarcely more scatter than the orbits of grade 1-4 actually used. This exemplifies once again the fact that even orbits of very low reliability for the individual elements may still give usable determinations of the "angular mass" $a^{3} P^{-2}$ and, therefore, also of 
TABLE I

Stars Showing Pronounced Deviation from the Main Sequence

\begin{tabular}{|c|c|c|c|c|c|c|c|}
\hline \multirow{3}{*}{$\frac{B-V}{-0.07}$} & \multirow{3}{*}{$\frac{M_{s}}{1.2}$} & \multirow{3}{*}{$\frac{\text { Star }}{15 \mathrm{~A}}$} & & & \multirow{3}{*}{$\frac{\text { Grade }}{4}$} & \multicolumn{2}{|c|}{ Spectral type } \\
\hline & & & \multicolumn{2}{|c|}{$\alpha, \delta(1900)$} & & Catalogue & Other \\
\hline & & & 01037 & +4643 & & B7 V & \\
\hline-0.02 & 2.5 & I $1336 \mathrm{~A}$ & 17403 & -3804 & 3 & B9 & \\
\hline-0.01 & -0.3 & A $1585, \kappa$ UMa A & 08568 & +4733 & 3 & B9n & $\mathrm{A} 0$ \\
\hline+0.01 & $2.7^{*}$ & $\mathrm{O} \Sigma 6 \mathrm{~A}$ & 00158 & +6627 & 3 & $B 8 V$ & A0 \\
\hline+0.06 & 3.2 & $\mathrm{~A} 2385 \mathrm{~A} \dagger$ & 15028 & +1850 & 4 & $\mathrm{~A} 2 \mathrm{~V}$ & A0 \\
\hline+0.11 & 3.1 & $\varphi 331,75$ Ori $A, a$ & 06116 & +0959 & 3 & $A 2 \mathrm{~V}$ & A2 \\
\hline+0.22 & 3.7 & A 1342 A & 09180 & -0925 & 3 & A2 & . \\
\hline+0.39 & 4.7 & A $337 \mathrm{~A}$ & 08123 & -0504 & 3 & $\mathrm{~F} 2$ & \\
\hline+0.42 & 0.9 & $0 \Sigma 517 \mathrm{~A}$ & 05083 & +0151 & 3 & A2 & \\
\hline+0.45 & 0.4 & $\beta 1240 \mathrm{~A}$ & 05322 & +3026 & 3 & G5 III & \\
\hline+0.45 & 5.0 & $\beta 64 \mathrm{~A}$ & 20403 & +1222 & 3 & $F 5 \mathrm{~V}$ & $F, G$ \\
\hline+0.49 & 2.0 & $\Sigma 2106 \mathrm{~A}$ & 16464 & +0935 & 4 & $\mathrm{dF} 4$ & \\
\hline+0.49 & $2.2^{*}$ & $\beta$ 151, $\beta$ Del A & 20328 & +1415 & 1 & F5 IV & $\mathrm{F5}$ \\
\hline+0.51 & 1.2 & $\varphi 326, \theta$ Ant $A$ & 09397 & -2719 & 3 & F7 V & dF7 \\
\hline+0.54 & 2.3 & A $2145 \mathrm{~A}$ & 10038 & +2049 & 3 & $\operatorname{gG5}(+\mathrm{A} 2)$ & $\mathrm{F} 5 \mathrm{n}(+\mathrm{A})$ \\
\hline+0.561 & 1.4 & $\Sigma 460 \mathrm{~A}$ & 03533 & +8025 & 4 & gG8 & \\
\hline+0.57 & 1.5 & B 430, $\psi$ Sgr A $\dagger$ & 19094 & -2526 & 2 & $F 8 \mathrm{~V}$ & $\mathrm{dF5}, \mathrm{F5}$ \\
\hline+0.64 & 3.1 & $\Sigma 2084, \zeta$ Her A $\neq$ & 16375 & +3147 & 1 & G0 IV & \\
\hline+0.65 & 1.8 & $\mathrm{O} \Sigma 2 \mathrm{~A}$ & 00082 & +2625 & 4 & F5 & \\
\hline+0.69 & $1.3^{*}$ & $\varepsilon$ Hya A & 08415 & +0647 & 1 & G0 III-IV & G0 III \\
\hline+0.75 & 3.5 & $\varphi 335 \mathrm{~A}, \mathrm{~B}$ & 08305 & -3215 & 3 & G5 & G5 IV-V \\
\hline+0.78 & 0.6 & Capella A & 05093 & +4554 & 1 & G5 III & G8 III, G0 III \\
\hline+0.795 & 1.9 & A $95 \mathrm{~A}$ & 19056 & -0735 & 3 & $F 9 I I I$ & G0 \\
\hline+0.80 & 2.6 & I $987 \mathrm{~A}$ & 16092 & -5327 & 3 & G5 V & \\
\hline+0.82 & 2.5 & $\Sigma 2367 \mathrm{~A}$ & 18374 & +3012 & 2 & G5 III & G5 \\
\hline+0.83 & 2.6 & A $2592 \mathrm{~A}$ & 17102 & -0942 & 4 & F5 & \\
\hline+0.84 & 3.4 & I $234 \mathrm{~A}$ & 13515 & -2702 & 4 & G5 & \\
\hline+0.88 & 1.1 & I $173 \mathrm{~A}$ & 10022 & -4653 & 4 & Ko IV & \\
\hline+0.88 & 2.2 & $\mathrm{Hu} 710 \mathrm{~A}$ & 07385 & -1650 & 3 & G5 III & G5 \\
\hline+0.90 & 1.3 & $\mathrm{R} 155 \mathrm{~A}$ & 10425 & -4854 & 3 & G5 III & \\
\hline+0.91 & 1.7 & $\Sigma 1687,35 \mathrm{Com} \mathrm{A}$ & 12484 & +2147 & 4 & G8 III & \\
\hline+0.91 & 1.7 & Hu 879, $\beta$ LMi A $\dagger$ & 10221 & +3713 & 3 & G8 III-IV & \\
\hline+0.96 & 3.5 & R $321 \mathrm{~A}$ & 20204 & -3744 & 3 & K2 IV-V & \\
\hline+0.99 & 4.2 & $17 \mathrm{~A}$ & 07146 & -4649 & 3 & $\mathrm{~K} 2 \mathrm{~V}$ & \\
\hline+1.00 & 3.2 & $\Sigma 73,36$ And A & 00496 & +2305 & 3 & $\operatorname{sgK1}$ & \\
\hline+1.01 & 1.2 & $\mathrm{O} \Sigma 359 \mathrm{~A}$ & 18314 & +2331 & 3 & gG8 & K0 \\
\hline+1.03 & $2.5^{*}$ & $\mathrm{AC} 2,95$ Cet $\mathrm{A}$ & 03132 & -0118 & 4 & K1 IV & K1 V, gK0 \\
\hline+1.07 & 0.0 & $\beta 1077, \alpha \mathrm{UMa} A \dagger$ & 10576 & +6217 & 2 & K0 III & K0 II-III \\
\hline+1.09 & $0.0^{*}$ & A 1377 A, B & 18317 & +5216 & 4 & K0 III & Ko \\
\hline+1.17 & 2.8 & A $108 \mathrm{~A}$ & 19417 & -0824 & 3 & K0 & \\
\hline+1.19 & -1.2 & $\lambda 179 \mathrm{~A}$ & 13252 & -3854 & 3 & G8 III & \\
\hline+1.39 & $0.2 *$ & $\beta 720,72$ Peg A & 23290 & +3047 & 4 & K4 III & $\mathbf{K} 2$ \\
\hline+1.43 & 7.4 & Don $91 \mathrm{~A}$ & 04582 & -2124 & 3 & $\mathrm{dM} 0.5$ & $\mathrm{dM} 1, \mathrm{M} 0$ \\
\hline+1.47 & 8.0 & Rst $1658 \mathrm{~A}$ & 12102 & -3032 & 4 & K5 & \\
\hline+1.49 & $7.9^{*}$ & Hu $115 \mathrm{~A}$ & 08085 & -1336 & 2 & $\mathrm{dMO}$ & \\
\hline
\end{tabular}

*Mean. †Spectroscopic binary? \$Perturbation suspected. 
TABLE II

Pairs for which $B-V$ is Avallable for Both Components

\begin{tabular}{|c|c|c|c|c|c|c|c|c|c|}
\hline \multirow{3}{*}{$\frac{\text { Star }}{\text { Jrb } 34^{*}}$} & \multirow{2}{*}{\multicolumn{2}{|c|}{$\alpha, \delta(1900)$}} & \multirow{2}{*}{\multicolumn{2}{|c|}{ Grade }} & \multirow[b]{2}{*}{$B-V$} & \multirow[b]{2}{*}{$M_{s}$} & \multicolumn{3}{|c|}{ Spectral type } \\
\hline & & & & & & & Cat. & Other & \\
\hline & 00127 & +4327 & 5 & $\begin{array}{l}\text { A } \\
\mathrm{B}\end{array}$ & $\begin{array}{l}+1.57 \\
+1.80\end{array}$ & $\begin{array}{r}9.4 \\
11.7\end{array}$ & $\begin{array}{l}\text { M3 V } \\
\text { M6 V }\end{array}$ & M1 V & SB \\
\hline $42 *$ & 00307 & +2927 & $\dagger$ & $\begin{array}{l}\text { A } \\
\text { B }\end{array}$ & $\begin{array}{l}+0.66 \\
+0.69\end{array}$ & $\begin{array}{l}5.1 \\
5.8\end{array}$ & $\begin{array}{l}\text { G2 V } \\
\text { G5 V }\end{array}$ & & \\
\hline 513,48 Cas & 01537 & +7025 & 1 & $\begin{array}{l}\text { A } \\
\text { B }\end{array}$ & $\begin{array}{l}+0.13 \\
+0.40\end{array}$ & $\begin{array}{l}2.5 \\
3.9\end{array}$ & A $4 \mathrm{~V}$ & & SB? \\
\hline$) \Sigma 149$ & 06302 & +2722 & 2 & $\begin{array}{l}\text { A } \\
\text { B }\end{array}$ & $\begin{array}{l}+0.57 \\
+0.85\end{array}$ & $\begin{array}{l}4.6 \\
5.9\end{array}$ & $\mathrm{dG} 2$ & & \\
\hline $2982,38 \mathrm{Gem}^{*}$ & 06490 & +1318 & 5 & $\begin{array}{l}\text { A } \\
\text { B }\end{array}$ & $\begin{array}{l}+0.31 \\
+0.72\end{array}$ & $\begin{array}{l}2.7 \\
5.1\end{array}$ & $\begin{array}{l}\text { F0 Vp } \\
\text { dG4 }\end{array}$ & & SB? \\
\hline$: 1321 *$ & 09078 & +5307 & 5 & $\begin{array}{l}\mathrm{A} \\
\mathrm{B}\end{array}$ & $\begin{array}{l}+1.38 \\
+1.34\end{array}$ & $\begin{array}{l}8.8 \\
8.9\end{array}$ & $\begin{array}{l}\text { MO V } \\
\text { MO V }\end{array}$ & & \\
\hline$\Sigma 231^{*}$ & 11056 & +3100 & $\dagger$ & $\begin{array}{l}\text { A } \\
\text { B }\end{array}$ & $\begin{array}{l}+1.34 \\
+1.48\end{array}$ & $\begin{array}{l}6.1 \\
7.4\end{array}$ & K9 V & $\begin{array}{l}\mathrm{dM} 1, \mathrm{dM} 0 \\
\mathrm{dM} 2\end{array}$ & \\
\hline : $1540,83 \mathrm{Leo}^{*}$ & 11217 & +0333 & 5 & $\begin{array}{l}\text { A } \\
\text { B }\end{array}$ & $\begin{array}{l}+0.79 \\
+1.03\end{array}$ & $\begin{array}{l}4.1 \\
5.0\end{array}$ & K0 IV & $\begin{array}{l}\mathrm{dK} 0 \\
\mathrm{dK} 5\end{array}$ & \\
\hline I N 28* & 14516 & -2058 & $\dagger$ & $\begin{array}{l}\text { A } \\
\text { B }\end{array}$ & $\begin{array}{l}+1.10 \\
+1.50\end{array}$ & $\begin{array}{l}6.7 \\
8.4\end{array}$ & $\begin{array}{l}\mathrm{dK} 5 \\
\mathrm{dM} 1\end{array}$ & & \\
\hline$: 2272,70 \mathrm{Oph}$ & 18004 & +0232 & 1 & $\begin{array}{l}\text { A } \\
\text { B }\end{array}$ & $\begin{array}{l}+0.78 \\
+1.15\end{array}$ & $\begin{array}{l}5.7 \\
7.1\end{array}$ & $\begin{array}{l}\text { K0 V } \\
\text { K4 V }\end{array}$ & $\mathrm{dK} 6, \mathrm{~K} 5 \mathrm{~V}$ & \\
\hline 2398 & 18417 & +5929 & $\S$ & $\begin{array}{l}\text { A } \\
\text { B }\end{array}$ & $\begin{array}{l}+1.54 \\
+1.59\end{array}$ & $\begin{array}{l}10.5 \\
11.1\end{array}$ & $\begin{array}{l}\mathrm{dM} 4 \\
\mathrm{dM} 5\end{array}$ & $\begin{array}{l}\mathrm{dM} 3.5, \mathrm{M} 2 \\
\mathrm{dM} 4\end{array}$ & \\
\hline 1648 & 18533 & +3246 & 2 & $\begin{array}{l}\text { A } \\
\text { B }\end{array}$ & $\begin{array}{l}+0.56 \\
+1.00\end{array}$ & $\begin{array}{l}4.5 \\
6.2\end{array}$ & Go V & & \\
\hline$\therefore 2758,61 \mathrm{Cyg}$ & 21024 & +3815 & 4 & $\begin{array}{l}\mathrm{Aa} \\
\mathrm{B}\end{array}$ & $\begin{array}{r}+1.17 \\
+1.38\end{array}$ & $\begin{array}{l}7.3 \\
8.0\end{array}$ & $\begin{array}{l}\text { K5 V } \\
\text { K7 V }\end{array}$ & & \\
\hline
\end{tabular}

* Not plotted in figure 1 .

$\uparrow$ Hyperbolic orbit.

tMisprinted +0.04 in catalogue of Blanco et al.

$\$$ Mean of Grade 4 elliptic and parabolic orbits.

the specific magnitude. However, in such cases it would seem more realistic to discard the illusory conventional elements and retain only $a^{3} P^{-2}$.

In Table I we list only the values of $M_{s}$ showing pronounced deviation from the main sequence, arranged in order of $B-V$ to facilitate identification. In many cases the deviation was to be expected $a$ priori.

Table II lists the few orbit pairs for which $B-V$ is available for both components. 
Spectral type

\begin{tabular}{|c|c|c|c|c|c|c|c|c|c|}
\hline \multirow{3}{*}{$\frac{\text { Star }}{\text { B } 1909 \mathrm{~A}}$} & \multicolumn{2}{|c|}{$\alpha, \delta(1900)$} & $P$ & $a$ & & $B-V$ & $M_{s}$ & \multirow{2}{*}{$\frac{\text { Cat. }}{\text { G2 IV }}$} & \multirow{2}{*}{$\frac{\text { Other }}{\text { G0, G4 IV }}$} \\
\hline & 00233 & -2053 & $5^{y} .625$ & 0.134 & A1 & +0.60 & $4.8^{*}$ & & \\
\hline & & & 11.25 & 0.214 & A1 & & $4.8^{*}$ & & \\
\hline \multirow{2}{*}{ A 939 A, B } & 01192 & +4505 & 64 & 0.27 & A3 & +0.34 & 4.6 & Fo & \\
\hline & & & 112.5 & 0.191 & A4 & & 3.1 & & \\
\hline Hwe 4 A & 01288 & -1244 & 144 & 0.919 & A3 & +0.64 & 6.3 & Go & \\
\hline \multirow[t]{2}{*}{$\mathrm{O} \Sigma 34 \mathrm{~A}$} & 01388 & +8023 & 165.4 & 0.35 & A3 & & 2.6 & A0 &. \\
\hline & & & 395.0 & 0.635 & A4 & & 2.7 & &. \\
\hline \multirow[t]{2}{*}{ A 1813 A } & 01562 & +3614 & 12.71 & 0.12 & A ?3 & +0.81 & $5.1^{*}$ & $G^{9} \mathrm{~V}$ & G5 \\
\hline & & & 25.8 & 0.19 & A ?3 & & $5.1^{*}$ & & \\
\hline \multirow[t]{3}{*}{ B 524 A } & 02474 & +3756 & 31.6 & 0.220 & $\mathrm{~A} 2$ & +0.42 & 2.2 & F4 V & F4 IV \\
\hline & & & 63.1 & 0.20 & $\mathrm{~A} 2$ & & 1.0 & & \\
\hline & & & 62.0 & 0.216 & $\mathrm{~A} 2$ & & -0.1 & & \\
\hline \multirow{2}{*}{ Ho $328 \mathrm{~A}$} & 04112 & +1926 & 60 & 0.348 & A3 & +0.405 & 4.0 & $F 4 V$ & dF5 \\
\hline & & & 225 & 0.47 & A4 & & 2.8 & & \\
\hline B $311 \mathrm{~A}$ & 04227 & -2418 & 175.7 & 1.25 & A 3 & +0.14 & 4.3 & $\mathrm{~A} 2$ & \\
\hline \multirow[t]{2}{*}{$\mathrm{Hu} 555 \mathrm{~A}$} & 04484 & +5156 & 101.32 & 0.215 & 4 & +0.45 & 3.3 & F8 & \\
\hline & & & 59.5 & 0.28 & 4 & & 4.6 & & \\
\hline \multirow[t]{2}{*}{ A $847 \mathrm{~B}$} & 05188 & -0058 & 24.68 & 0.1887 & A3 & $(+0.51) \dagger$ & 4.0 & $F 8 V$ & \\
\hline & & & 49.36 & 0.3320 & $\mathrm{~A} 3$ & & 4.3 & & \\
\hline A 1959 A, B & 06567 & +3949 & 40.2 & 0.27 & A ?3 & & 5.9 & & \\
\hline \multirow[t]{2}{*}{ A $1101 \mathrm{~A}$} & 14109 & +1046 & 36.0 & 0.28 & A 3 & +0.86 & 6.3 & K0 & \\
\hline & & & 73.0 & 0.30 & A3 & & 5.4 & & \\
\hline \multirow[t]{2}{*}{$\mathrm{Hu} 1266 \mathrm{~A}$} & 14122 & +3400 & 38.9 & 0.16 & A3 & & 5.0 & K0 & \\
\hline & & & 37 & 0.356 & A3 & & 6.8 & & \\
\hline A 2983 A, B & 14444 & +1038 & 9.85 & 0.1699 & $\mathrm{~A} 2$ & +0.87 & 6.5 & $\mathrm{~K} 2 \mathrm{~V}$ & G5 \\
\hline & & & 9.92 & 0.165 & $\mathrm{~A} 2$ & & 6.4 & & \\
\hline & & & 19.8 & 0.155 & $\mathrm{~A} 2$ & & 5.3 & & \\
\hline $\mathrm{Hu} 580 \mathrm{~A}$ & 15371 & +2000 & 11.07 & 0.117 & A ?1 & +0.039 & $1.6^{*}$ & A1 V & A2 \\
\hline & & & 22.14 & 0.210 & A ? 1 & & $1.8^{*}$ & & \\
\hline A $2080 \mathrm{~A}$ & 15496 & +1717 & 90 & 0.31 & A ?3 & & 3.8 & $\mathrm{~F} 2$ & \\
\hline Hu 1176 A, B & 17045 & +3604 & 16.08 & 0.167 & A2 & +0.31 & 2.8 & A5 & \\
\hline & & & 8.04 & 0.2285 & A2 & & 4.5 & & \\
\hline & & & 8.159 & 0.116 & $\mathrm{~A} 2$ & & 3.0 & & \\
\hline A $2192 \mathrm{~A}$ & 18508 & +0319 & 135 & 0.267 & A4 & +0.155 & 2.3 & $B 9 V$ & A2 \\
\hline & & & 225 & 0.350 & A4 & & 2.2 & & \\
\hline & & & 85.4 & 0.49 & A3 & & 4.3 & & \\
\hline A 604 A & 19524 & +0457 & 146.67 & 0.2051 & A4 & +0.57 & 3.0 & $\mathrm{dF} 8$ & \\
\hline A $617 \mathrm{~A}, \mathrm{~B} \dagger$ & 21165 & +0955 & 12.20 & 0.184 & A ?3 & +0.53 & 4.7 & $F 8 \mathrm{~V}$ & F7 \\
\hline $\mathrm{Hu} 280 \mathrm{~A}$ & 21373 & +0527 & 112.1 & 0.1907 & A3 & & 2.5 & & \\
\hline & & & 77.84 & 0.260 & $\mathrm{~A} 3$ & & 3.7 & & \\
\hline$\varphi 283 \mathrm{~A}$ & 21395 & -5808 & 6.32 & 0.2335 & $\mathrm{~A} 2$ & +1.32 & $8.1^{*}$ & $\mathbf{M}$ & K7 V \\
\hline & & & 12.65 & 0.308 & $\mathrm{~A} 2$ & & $7.7^{*}$ & & \\
\hline$\varphi 307 \mathrm{~A}, \mathrm{~B}$ & 21511 & -5528 & 6.094 & 0.127 & $\mathrm{~A} 2$ & +0.29 & $2.5^{*}$ & F0 IV & Fo \\
\hline & & & 12.242 & 0.160 & A2 & & $2.0^{*}$ & & \\
\hline Ho 482 A, B & 22466 & +2552 & 55.8 & 0.30 & $\mathrm{~A} 2$ & +0.23 & 3.8 & $A I V$ & A3 \\
\hline & & & 102.3 & 0.225 & A3 & & 2.3 & & \\
\hline & & & 243.26 & 0.422 & A3 & & 2.4 & & \\
\hline B $182 \mathrm{~A}$ & 23119 & -1422 & 90 & 0.48 & A ?4 & +0.47 & 5.2 & G0 VI & $\mathrm{sdF} 8, \mathrm{sdG} 2$ \\
\hline
\end{tabular}


In Table III we give the values of $M_{s}$ and $B-V$ for "ambiguous" orbits arising from uncertainty of quadrant interpretation. It will be noticed that in the classical type of ambiguity (e.g., B 1909) where the period of one orbit is approximately double that of the other and the eccentricities are small and large respectively, the values of $M_{s}$ do not differ greatly and therefore cannot be used as a discriminant. In some other cases, however, the values of $M_{s}$ may differ sufficiently to enable a choice to be made.

Note added: Worley has pointed out that the $B-V$ values for the components of $\beta 513, O \Sigma 149$ and $\beta 648$ (Table II) quoted from the catalogue of Blanco et al. were not observed directly but derived from the combined values of $+0.17,+0.64$ and +0.59 , respectively.

\section{REFERENCES}

Alexander, J. B. 1970, Mon. Notes astr. Soc. South Africa, 29, 44.

Blanco, V. M., Demers, S., Douglass, G. G. and FitzGerald, M. P. 1968, Pub. U.S. Nav. Obs., 2nd ser., 21.

Finsen, W. S. and Worley, C. E. 1970, Republ. Obs. Johannesburg Circ., 7, 203.

Harris, D. L., Strand, K. Aa. and Worley, C. E. 1963, Empirical Data on Stellar Masses, Luminosities, and Radii in Basic Astronomical Data, ed. K. Aa. Strand, Univ. of Chicago Press, p. 285.

Hertzsprung, E. 1964, J. des Obs., 47, 31.

Knipe, G. F. G. 1969, Republ. Obs. Johannesburg Circ., 7, 197. 1970, ibid., 7, 260.

Smak, J. 1967, Acta astr., 17, 213.

\section{Discussion}

Strand remarked that this idea of Hertzsprung's had first appeared in the second of his first two professional papers, published in the Zeitschrift für wissenschaftliche Photographie in 1905 and 1907. In that paper he used this method to discuss the luminosities of the components of Capella. Hertzsprung apparently had forgotten this in his 1964 paper where he referred to a paper published in 1911 .

\section{Session 3. Multiple Systems}

In his introductory talk, A. Poveda divided the subject into its morphological, dynamical, and cosmological aspects. The idea that runaway stars originate from binaries, due to an explosive mass loss of the primary liberating the secondary, was so difficult to understand that another explanation should be found. 